\title{
La identidad profesional interpelada. De la presencia de otros, de trayectorias, experiencias y 'huellas' en profesores de Escuela Media
}

\section{Questioned professional identity. From the presence of others, paths, experiences and 'fingerprints' of teachers in High School}

\author{
Mgter. Patricia Delgado ${ }^{1}$ - Mgter. Mariana Ojeda ${ }^{2}$ - Lic. Claudio Nuñez ${ }^{3}$
}

Fecha de recepción: noviembre 2010

Fecha de aceptación y versión final: mayo 2011

Resumen: Desde una línea de investigación sobre el trabajo del docente en escuelas secundarias de la provincia del Chaco (Argentina), se aborda la problemática de la construcción de la identidad profesional focalizando las representaciones que construyeron de sí mismos un grupo de docentes a lo largo del proceso de formación.

La concepción de la identidad del docente como un constructo conformado a lo largo de una trayectoria profesional es consistente con un abordaje metodológico de tipo biográfico - narrativo que recupera en el presente un pasado en el que se entraman experiencias públicas y privadas, colectivas e individuales, personales y profesionales.

Se desarrollan los componentes configuradores de esa identidad que le otorga sentido al trabajo, y que son reconocidos por los propios docentes como significativos, señalando además la importancia de ciertas experiencias en el trayecto que han dejado huellas profundas en su formación, marcando los orígenes de aquellas representaciones.

Se destaca la relevancia de reflexionar en torno a estas cuestiones debido al momento de crisis de sentido de la escuela Media en nuestra sociedad, que afecta a algunos de los núcleos esenciales de la identidad profesional, interpelándolos y amenazando su continuidad.

Palabras claves: identidad profesional - identidad del docente de nivel medio - formación docente.

Abstract: This is a line of investigation into the work of teachers in High Schools in the province of Chaco (Argentina), which deals with the construction of professional identity, focusing on the representations that they built from themselves as a group of teachers throughout the process training.

The components of the identity of the teacher as a construct formed are designed along a career pathway. This is consistent with a kind of biographical study methodology - narrative, which reflects a past in the present in which experiences are entering in their public and private, collective and individual, personal and professional lives.

The components are developed into parameters of that identity, that gives meaning to work, and are recognized by the teachers themselves as significant, noting the importance of certain experiences on the journey that has left deep traces in their formation, marking the origins of those representations.

This work highlights the importance of reflecting on these issues, due to the fact of the sense of crisis that high school in our society, affects some of the essential nucleus of professional identity, questioning and threatening their survival.

Key words: professional identity - identity of the high school teachers - teacher training.

\footnotetext{
${ }^{1}$ Facultad de Humanidades - UNNE - correo electrónico: pmadelgado@yahoo.com.ar

${ }^{2}$ Facultad de Humanidades - UNNE - correo electrónico: marianaojeda@ gigared.com

${ }^{3}$ Facultad de Humanidades - UNNE - correo electrónico: claudionunez26@ hotmail.com
} 


\section{La construcción de la identidad profesional de los docentes secundarios en el marco de nuestra investigación ${ }^{4}$}

A partir de nuestras investigaciones referidas al trabajo del profesor de enseñanza media, presentamos en el artículo algunas reflexiones referidas a la problemática de la construcción de su identidad profesional. En particular nos interesa describir algunos elementos que la componen y comprender cómo fueron adquiridos en el transcurso de su formación, entendida como un proceso de desarrollo personal y profesional (Ferry, 1997).

En este sentido, definimos a la identidad del docente como un constructo conformado a lo largo de una trayectoria profesional, a través de un entramado de experiencias formativas que tienen lugar en distintos ámbitos de la vida de los sujetos: personal, académico, y laboral. Esa representación de sí como profesional otorga al individuo un sentido subjetivo de mismidad y continuidad, a pesar de los cambios que se van sucediendo en el curso de su existencia (Erikson, 1980, cit. por Bolivar, 1999).

La identidad incluye siempre elementos de una cultura profesional, que interactúan con otros contenidos más personales y singulares. Por cultura profesional entendemos un marco de comprensión compartido de la realidad profesional, que permite a los profesores reconocerse como pertenecientes a un mismo colectivo, en torno a una actividad que los diferencia de otros grupos profesionales (Rivas Flores, 2000). Se adquiere en el proceso de socialización como un legado histórico, fruto de la transmisión de modelos y también como una transacción en las relaciones entre los actores sociales del cuerpo (Dubar, 1991 cit. por Lang, 2005), lo que implica reconocer un plano de apropiación individual de la profesión y un plano social de producción y reproducción de la misma.

Consideramos que un abordaje metodológico adecuado para comprender el proceso de configuración de la identidad profesional es la reconstrucción de las biografías profesionales a través de la narrativa ${ }^{5}$, en tanto permite a los profesores "poner afuera" un conjunto de acciones y pensamientos propios para poder mirarlos con cierta distancia, analizando las decisiones que toman, cómo realizan sus tareas, con quiénes y por quiénes están condicionados, pudiendo así identificar las condiciones de producción del trabajo como parte constituyente de su identidad y de su cultura (Veiravé, Ojeda, Núñez, Delgado; 2006).

Por eso hemos realizado series de entrevistas en profundidad a diez profesores de tres escuelas medias de la Provincia del Chaco, situadas en contextos sociales diferentes: el centro de la ciudad capital, un barrio periférico y una pequeña localidad del interior de la provincia. En esas entrevistas los docentes han narrado y caracterizado distintos momentos de su carrera profesional: la elección de la profesión, la formación inicial y continua, las experiencias laborales, las personas significativas,

\footnotetext{
${ }^{4}$ Este trabajo forma parte de una línea de investigación que se desarrolla desde 2003 en el Instituto de Ciencias de la Educación de la Facultad de Humanidades de la Universidad Nacional del Nordeste, a través de sucesivos proyectos acreditados por la institución. El primero, denominado "Vida y trabajo de los profesores de enseñanza secundaria. Institución y profesión docente desde una perspectiva intercultural"; el segundo, "Escuela media y Trabajo Docente en la Provincia del Chaco. Dimensiones políticas, institucionales, culturales y sociales en la configuración del trabajo de los profesores y de las instituciones educativas", en curso actualmente. Ambas investigaciones dirigidas por la Prof. Delfina Veiravé.

5 Distinguimos las biografías profesionales de las de carácter personal, en tanto aluden a relatos que provocan la reflexión y el análisis de su propia vida en el entorno profesional, aunque la trayectoria profesional esté íntimamente ligada a la vida privada de los sujetos.
} 
las condiciones de trabajo en las instituciones, las relaciones con los colegas, directivos, alumnos, y tutores, entre otros aspectos.

Metodológicamente consideramos que la narración y descripción de hechos del pasado que hacen los profesores al reconstruir sus biografías profesionales, suponen un recuerdo selectivo y subjetivo de circunstancias, sucesos, experiencias, atravesadas por pensamientos y sentimientos que los interpretan (Kornblit, 2004). Es así como en los relatos de los sujetos podemos advertir la emergencia de algunos componentes centrales de la identidad profesional en su propio devenir, entramando planos individuales y colectivos, personales y profesionales, públicos y privados.

Es decir, en el relato biográfico encontramos "huellas" o anclajes de esa identidad profesional que inferimos a partir del discurso de los sujetos. En este trabajo buscamos comprender la relación entre algunos de esos aspectos que se configuraron en el pasado y que están presentes hoy en su lectura de la realidad y de sí mismos. En este sentido nos preguntamos: ¿qué elementos componen la identidad profesional de los docentes de nivel medio estudiados? ¿cómo van adquiriéndolos en el marco de las experiencias individuales y colectivas, personales y profesionales acontecidas en el transcurso de la formación?

Estos interrogantes asumen relevancia en un escenario caracterizado por una crisis de sentido de la Escuela Media y del rol de los profesores que trabajan en ella. En el ida y vuelta del pasado al presente, encontramos que algunos de los fundamentos de la identidad se han ido erosionando como resultado de la convergencia de fenómenos socioculturales y políticos más amplios, que producen un quiebre de ciertas tradiciones escolares, cambios en la dinámica de relaciones, las prácticas y las significaciones. Responder entonces a los interrogantes iniciales nos ha llevado a reconocer en los profesores procesos identitarios en tensión, que se reflejan en una pérdida de la capacidad para reconocerse a sí mismos y el sentido de la tarea que realizan, en el grado de compromiso con un proyecto compartido, en el sentido de una identidad comunitaria que permite lograr resultados que otorguen reconocimiento social y satisfacción individual frente a la tarea de educar. Son éstas las cuestiones que abordaremos en los apartados siguientes.

\section{Notas definitorias de la identidad profesional en un grupo de profesores de nivel medio}

La lectura de las narrativas de los profesores nos permitió reconocer algunos rasgos decisivos en la definición de la identidad profesional, los que operan no sólo como elementos de identificación con un colectivo profesional, sino también como criterios de clasificación y de calificación de los colegas que pertenecen al mismo, estableciendo diferencias entre sectores y grupos. Esos rasgos emergentes del análisis comparativo nos posibilitaron la construcción de las siguientes categorías de análisis:

\section{Vocación y compromiso}

Los profesores reconocen en sí mismos una disposición natural para ejercer la docencia, descubierta en diferentes momentos: antes de elegir la carrera, durante la formación inicial o trabajando ya en el sistema educativo. Es lo que ellos expresan como "llevar la docencia en la sangre", o "ser docente por naturaleza". Es decir, la 
vocación como una aptitud innata que se expresa sobre todo en el placer de enseñar. En este sentido, una profesora señala:

“... creo que desde que nací descubrí mi vocación porque siempre dije que iba a enseñar... Amo la docencia y disfruto lo que hago, que es lo que siempre quise hacer" (J.)

Otros profesores relatan haber descubierto su vocación docente ya en el ejercicio de la profesión. Para ellos, el ingreso a la carrera del profesorado no fue la primera opción, sino una alternativa viable relacionada con aquélla. Estas situaciones ilustran de qué manera el ámbito privado incide en la elección profesional, porque en cada caso, situaciones personales familiares (casamiento, migraciones, necesidades económicas) los condujeron a ingresar a la formación y al trabajo docente.

La vocación se asocia también al sentido del compromiso: con la tarea educativa, con los alumnos, con el establecimiento. Constituye uno de los criterios más utilizados para marcar diferencias entre grupos de profesores: los que se comprometen y "los que dan clases y se van" (tal como dicen muchos de los entrevistados). Esta idea se plantea en estos términos o caracterizando a la docencia como "apostolado".

Estos sentidos (y sus matices) asociados al componente vocacional de la docencia, remiten a una de las tradiciones de la formación docente, definidas por Davini como "...configuraciones de pensamiento y de acción que, construidas históricamente, se mantienen a lo largo del tiempo en cuanto están institucionalizadas e incorporadas a las prácticas y a la conciencia de los sujetos" (Davini, 1995: 20). Nos referimos a la tradición normalizadora-disciplinadora, con su énfasis en el papel moralizador y socializador del docente, concebido como ejemplo o modelo que asume su tarea desinteresadamente, con un fuerte compromiso y entrega personal.

No obstante, creemos que esos sentidos originales aparecen resignificados. Acordamos con Almandoz y Cigliutti cuando señalan:

"...la docencia ha privilegiado el compromiso afectivo con los alumnos (en lugar del disciplinamiento), a quienes entrega innumerables esfuerzos, y el rescate del discurso vocacionista que estructura la unidad de la categoría laboral, pero dirigido a la tarea y no al servicio del proyecto del Estado. Con ello, las improntas de origen se sostienen dentro de una escuela en crisis y con un trabajo docente en situación de riesgo" (Almandoz y Cigliutti, 1992, en Davini, op.cit:28)

Es decir, el hecho de que los docentes reconozcan y valoren como componentes de la identidad profesional la vocación y el compromiso, no significa que adscriban a determinada tradición en todos sus términos. En este sentido, Tenti (2005) reconoce que cuando se trata de servicios personales, quien los presta sólo puede realizar bien su tarea si combina un fuerte compromiso ético y afectivo hacia los sujetos con quienes trabaja, con la excelencia en términos de competencia y dominio de saberes especializados; aunque también plantea la necesidad de revisar la idea de la vocación como predisposición innata reconociendo que se trata de una construcción histórica y cultural, o la que la asocia con la consagración social y el desinterés.

\section{El docente como formador}

En las entrevistas, la docencia asume un sentido que trasciende al de la enseñanza como proceso de transmisión de saberes. Remite a la formación de los alumnos como personas, que se asemeja de algún modo a una tarea "artesanal": moldear al alumno y ser "modelo" para ellos. Los docentes se definen como educadores, además de ser profesores de "X" disciplina. Intentan formar en valores y actitudes, por eso priorizan 
la atención a los alumnos, establecer un vínculo con ellos, lo que implica a veces contener y otras poner límites.

Ser docentes significa entonces presentarse al otro como ejemplo de comportamiento, portador y transmisor de valores morales considerados importantes, con la deliberada finalidad de generar una formación integral en el otro. En esta idea coinciden todos. Las variaciones que encontramos en los profesores entrevistados tienen que ver con los modelos pedagógicos a los que adhieren. Por ejemplo, una profesora expresa ser "de la guardia vieja", en el sentido de tradicional, de valorizar la lectura, el estudio, la memoria, la presentación impecable del profesor; mientras que otros se definen como más innovadores o planteando una relación con los alumnos en términos de amistad.

Más allá de estas diferencias, la idea fuerte del docente como modelo formador de valores remite también a la tradición normalizadora-disciplinadora, mencionada en el punto anterior.

\section{La disciplina de enseñanza}

Los campos disciplinares y profesionales tienen un poder decisivo en la configuración de la identidad de los profesores. En la mayoría de los casos la elección de la disciplina precede a la elección de la docencia. Es decir, se manifiesta primero el interés por la disciplina, mientras que la disposición a la docencia se va constituyendo en el proceso de formación inicial y en la práctica laboral. De allí la elección del nivel, ya que en la Escuela Media se es docente de una disciplina específica.

Además, la inserción en los establecimientos depende de esta condición. Los profesores se agrupan en Departamentos de materias afines, interactúan más con colegas del área disciplinar, con quienes deben planificar, articular contenidos, organizar actos, exhibiciones, etc. $\mathrm{Y}$, según su especialidad, adquieren determinado status en el colegio, como lo ilustran las siguientes citas:

"(los contables) éramos una especie de elite, porque era la Escuela de Comercio... los de otras materias complementarias se sentían en inferioridad de condiciones respecto de nosotros..." (N.)

“...siempre me peleo con los directivos, nunca nos dan unas glosas para hacer en los actos...siempre nos dan las sillas para acomodar...creo que esto ocurre porque siempre les dan más importancia a otras materias como Matemática, Lengua, Historia y Geografía..." (V. profesora de Educación Física)

Los profesores reconocen en sí mismos características personales relacionadas con la disciplina elegida. Por ejemplo, una profesora de educación física dice "yo soy aventurera" mientras cuenta los viajes o campamentos que hacía con los alumnos; el profesor de matemática dice "yo soy de explicar", y relata cómo expone diferentes demostraciones a los alumnos; una profesora de historia expresa "me apasiona la lectura y conocer el origen de las cosas". En este sentido, manifiestan su representación de sí mismos en el "yo soy" profesional y personal, vinculando estas dos dimensiones.

La importancia que asume la disciplina en la identidad y en la experiencia de trabajo de los profesores remite a otra de las tradiciones que tuvo gran influencia en la formación de los docentes de nivel medio en nuestro país: la tradición académica. Ésta se funda en dos ideas centrales: a) lo esencial en la formación y acción de los docentes es que tengan un sólido conocimiento de la materia que enseñan, y b) la 
formación pedagógica es débil, superficial e innecesaria, pues el saber hacer se adquiere en la experiencia práctica (Davini, 1995)

El hecho de que los profesores entrevistados coincidan en señalar que la formación inicial (en la universidad o el instituto terciario) les ha brindado conocimientos teóricos, sobre todo los de la disciplina a enseñar, pero que el conocimiento del "oficio" es dado por la práctica, refleja la vigencia de esta tradición en la formación docente.

\section{El trabajo con adolescentes}

Al gusto por la enseñanza de la disciplina se une la preferencia por trabajar con adolescentes y jóvenes, cuestión que marca la diferencia con otros colectivos docentes de otros niveles educativos. Es en la relación con los alumnos donde encuentran las mayores gratificaciones a pesar de los problemas que les plantea su labor cotidiana. Encontramos expresiones que lo manifiestan:

"siempre dije que iba a enseñar, pero yo no quería ser maestra, quería trabajar con adolescentes..." (J.)

“... lo mío era relacionarme con los adolescentes. Para mí era lo más increíble que podía haber y me llevaba bien con ellos, llegamos a tener una relación de amistad con algunos..." (ME)

El lugar que ocupa el vínculo con estos alumnos que se encuentran en este ciclo de vida en particular colabora en la delimitación de la identidad. La problemática adolescente atribuye un sentido específico a las relaciones pedagógicas y sociales: el manejo del grupo, de la disciplina, la atención a las circunstancias psico-afectivas y sociales propias de este ciclo. A estas se suman las problemáticas sociales y culturales actuales configurando un estado de situación especial que demanda otro tipo de atención. Como lo explica una profesora:

"Lo único que te puedo decir es que los adolescentes están pidiendo a gritos atención porque han perdido la atención de la casa, está quebrada la familia y necesitan una oreja que los escuche. Esa es la problemática más acuciante en todas las escuelas" (J)

\section{Las características del Nivel Medio}

La escuela media constituye un tramo de formación de carácter propedéutico, ya sea para continuar estudios superiores o incorporarse en el mercado de trabajo. Se distingue de otros niveles educativos por reunir ciertas características como: la fragmentación del currículum en materias organizadas con criterio disciplinar, la especialización de los profesores en disciplinas específicas, el trabajo con adolescentes y jóvenes, la organización departamental, la designación de los profesores por horas cátedra, en general la pertenencia de los docentes a distintas instituciones. Esta organización del trabajo docente refuerza la tendencia al aislamiento y la cultura individualista, como lo ilustra esta cita:

“...creo que ese es el mayor de los fracasos que he vivido en la docencia, el no poder conciliar con mis colegas...no hay coordinación, ninguno quiere sumarse a nada que sea ocupar más horas, se limitan a su clase y nada más" (J.) 


\section{La titulación que acredita el rol}

Requisito que acredita la inserción profesional en el ámbito laboral, en la mayoría de los casos se presenta como una condición para validar, legitimar el lugar que el docente ocupa en el sistema, y también como condición para garantizar la estabilidad laboral. Uno de los docentes entrevistados no tiene título docente y no lo vive como un "estigma", aunque sabe que pueden desplazarlo de sus horas y perder su trabajo. Otra profesora nos cuenta que hizo el profesorado aunque sus horas eran titulares, porque sentía que "no estaba a la altura de las circunstancias", que su hacer no tenía un respaldo académico.

En este sentido, el haberse formado y egresado de una institución de formación docente que posiciona socialmente y habilita en la profesión constituye también un rasgo identitario. Aunque en algunos casos no haya constituido una primera opción vocacional y profesional es una condición que hace a la identidad profesional y por eso quizás constituye otro de los criterios de diferenciación que utilizan los docentes para distinguirse entre si. En este sentido, un profesor plantea:

"... sabés cual es el problema? que solamente nos comprometimos los que somos profesores con título, antes éramos pocos profesores con título y había maestros, podían dar técnicos, cualquiera enseñaba acá... entonces no había compromiso de parte de la otra gente, para nosotros era nuestra carrera, para ellos era solamente una salida laboral momentánea... en esta escuela, hace tres o cuatro años empiezan a llegar profesores con título ..." (C)

De esta serie de componentes de la identidad profesional, notamos que los cuatro primeros definen a la profesión en un sentido intrínseco a ella, es decir desde lo particular y propio de la tarea, mientras que los dos últimos refieren a las condiciones de la estructura y la organización socio-laboral, que estarían operando como condiciones extrínsecas en tanto configuran esa tarea desde fuera, desde el sistema que la regula.

\section{Los anclajes de la identidad profesional de los docentes}

Identificamos algunos sucesos en la trayectoria profesional de los docentes que, de acuerdo al modo en que los sujetos los significan y nuestras propias interpretaciones, consideramos han incidido profundamente en la conformación de los rasgos de la identidad profesional.

Encontramos en las narrativas experiencias significativas de formación que exceden los ámbitos de formación docente inicial y contínua. En este sentido, autores como Diker y Terigi (1997) y Davini (1995) reconocen como ámbitos de formación docente a la trayectoria escolar previa o biografía escolar y la socialización profesional $^{6}$ que se desarrolla en las escuelas y los colegios donde se desempeñan. Observamos entonces que la formación inicial acontecida en el profesorado se convierte en uno de los ámbitos de anclaje de la identidad, no el exclusivo.

Todos estos ámbitos de experiencia se entrelazan en los recuerdos, conformando un entramado de espacios y tiempos que incluye también los propios de la trayectoria

\footnotetext{
${ }^{6} \mathrm{Al}$ respecto las autoras entienden que la trayectoria escolar previa de los sujetos (como alumnos de la escuela primaria y secundaria) es un período de socialización profesional en el rol porque interiorizan modelos de aprendizaje y rutinas escolar es que, de alguna manera, se actualizan cada vez que se desempeñan en el rol de enseñantes. La socialización profesional alude a la inserción del docente principiante o novel en la escuela como lugar de trabajo que a la vez es ámbito de formación porque adquirirá las herramientas para afrontar la práctica real y cotidiana.
} 
de vida personal, es decir, la ineludible consideración de los ámbitos familiares y socio-políticos que van configurando las decisiones de las personas. En este devenir de la vida personal y profesional encontramos aspectos en los que podemos localizar anclajes de la identidad.

\section{Los rasgos de los otros significativos que se incorporaron en los procesos de identificación ${ }^{7}$ durante la biografía escolar y la formación inicial.}

Un aspecto sobresaliente en los relatos fue la "marca" que imprimieron ciertas personas que los acercaron a la docencia, siendo referentes o modelos a imitar. En el sentido de Berger y Luckman (1991) los otros significantes actúan como andamiajes que aproximan u ofrecen modelos de comportamiento y marcos de comprensión de la realidad. Entre los otros significativos se mencionan padres y algunos familiares docentes, profesores de nivel medio y en menor medida de la primaria, profesores de la institución de formación inicial, y algunos colegas en las primeras experiencias laborales.

Respecto de las figuras familiares significativas, de ellas recuperan sobre todo algunos valores (el esfuerzo, la rectitud, la autoexigencia), y el estímulo/mandato de estudiar. Algunos tuvieron padres o madres docentes, que transmitieron imágenes sobre la profesión y el trabajo. Respecto de sus profesores de nivel medio y los del profesorado, aparecen figuras significativas operando como modelos y contramodelos. Caracterizan a los profesores modelo como "buenos" profesionales y "buenas" personas, habiendo podido crear con ellos un vínculo fundado en la admiración por la forma de enseñar y el saber que demostraban, la valoración de la exigencia que tuvieron con ellos, el acercamiento y la ayuda personal que brindaban. De estos modelos - a quienes llaman, por ejemplo, "personajes de ese calibre", recuperan la responsabilidad, la rectitud, la exigencia, la pasión por el saber y la enseñanza, la humanidad, los valores morales, la presentación personal pulcra, el diálogo y la atención personal al alumno, características que componen su propia definición del sí mismo profesional. El siguiente fragmento ilustra esta idea:

"por ejemplo en gramática... el profesor S. .. yo lo admiraba, la forma en que nos enseñaba y la forma en que nos exigía ... cómo nos explicaba ... es lo que yo trato de trasladar a los chicos." (M.E.)

De los colegas que los ayudaron en las primeras experiencias laborales, destacan sobre todo el "saber hacer" y la generosidad de enseñarle el "oficio":

"yo me acerque a mis grandes profesoras de Lengua que tenía en la escuela...me arrimé a ellas para aprender...vieron mis ganas y me formaron. Yo tengo esa escuela de grandes profesores...la Sra. M., iba a la casa y ella me enseñaba a planificar...Yo nunca había tomado examen oral a los alumnos, la primera vez que estuve en una mesa de examen dije: ¿qué estoy haciendo acá? No sabía cómo se completaba un libro de actas, no sabía cómo se llenaba un libro de temas, esas cosas las fui aprendiendo de ella... aprendí todo, como un aprendiz al lado de su maestro" (J.)

Estos otros significativos no sólo permitieron a los profesores entrevistados adquirir seguridad y confianza en sí mismos sino que también marcaron fuertemente la identificación con la disciplina que enseñaban, lo que permitió la introducción y la formación en el campo disciplinar de elección. También influyeron en el modo de plantear la relación con el alumno, dependiendo esto del modelo pedagógico adoptado

\footnotetext{
7 "La identificación es uno de los mecanismos psíquicos, por el cual el individuo interioriza las características o los diferentes aspectos buenos o malos del OTRO" (Abraham, 1998: 11).
} 
por cada profesor. En relación a este punto, uno de los aspectos que identifican a los profesores que actuaron como contramodelos es esta ausencia de un vínculo personal con el alumno:

“...viví experiencias en la escuela $N$. con profesores de Lengua que no permitían ese acercamiento del docente al alumno, ellos en el estrado y nosotros abajo...entonces yo dije: algún día voy a ser profesora en Letras y voy a ser amiga de mis alumnos...Me hice esa promesa, tener diálogo y ser la oreja de los alumnos, porque nosotros en la escuela $N$. sufrimos. A los profesores los admiraba como docentes no como seres humanos o sea les faltaba la otra parte para que fueran mejores...por eso hoy tengo la relación que tengo con mis alumnos."(J.)

Estos otros significativos ofrecen modos de ser y de no ser docentes con los cuales conformar la propia identidad profesional. En un proceso de deliberación que se hace visible en ocasión del relato biográfico, manifiestan la manera en que se produce dicha identificación "es inconsciente, uno lo va mamando, se le va metiendo... uno va tomando ese ejemplo" (ME). A partir de un proceso relacional, los docentes internalizan características personales y profesionales con las que se identifican, es decir, que desean poseer o poseen y se ven proyectadas en ellos.

\section{La adquisición del rol profesional en el entramado de experiencias de la formación inicial y de los primeros desempeños: entre la teoría y la práctica docente}

Al reflexionar sobre sus experiencias formativas durante el cursado del profesorado (en la universidad 0 el instituto terciario) o durante sus primeras experiencias laborales, los docentes coinciden en una idea que podríamos resumir en estos términos: la formación inicial brinda los conocimientos teóricos, sobre todo los de la disciplina a enseñar, pero el conocimiento del oficio es dado por la práctica y la imitación de modelos (otros significativos). Lo ilustramos en las siguientes citas:

"(en el Instituto) te dan la base, la teoría, pero no te preparan para ser docente, eso te lo da la experiencia" (V)

"...la Facultad no nos dio herramientas para la realidad de la escuela secundaria, para mí fue como empezar de cero...” (J.)

“...el profesorado no nos prepara para estar frente al alumno, sí nos prepararon en cuanto a conocimientos, pero no para situaciones frente a alumnos, el trato con los adolescentes, los problemas que se presentan..." (H.)

“...la formación en el instituto fue bastante exigente en el contenido, (pero) la carrera no me preparó para enseñar...entre lo que yo me preparé y lo que fui adquiriendo en la docente, me fui haciendo...o sea, no nos aportó la Didáctica..." (C.)

A esta distancia entre la formación de fuerte carácter teórico en las instituciones formadoras y el ejercicio profesional, los docentes atribuyen el "shock" que les produjeron las primeras situaciones "frente a alumnos", como ellos definen a las situaciones de práctica docente, aunque este fenómeno aparece en algunas de las entrevistas, no en todos los casos. Conviene aclarar que sólo uno de los profesores entrevistados comenzó su experiencia laboral con título, mientras que los demás estudiaron y trabajaron simultáneamente

Identificamos en la formación inicial una fuerte impronta de formación teóricaacadémica mientras que el conocimiento del oficio se genera en la práctica del mismo, que exige el desarrollo de un saber hacer en tanto "profesores de secundario". Las 
primeras experiencias laborales colaboran en la conformación y confirmación del rol al producir aprendizajes de "ajuste situacional" a la cultura profesional y laboral (Bolivar, 1999:95). En este sentido, mostrar idoneidad en el conocimiento y la transmisión de los contenidos, así como en el manejo del grupo de clase; es decir, desempeñar estas habilidades con seguridad en las situaciones de práctica, permite confirmar que "sí" pueden ser docentes.

Durante el ejercicio docente se plantean prácticas profesionales para las cuales "no estaban preparados". Los docentes buscan soluciones a los problemas de la práctica preguntando a colegas más experimentados, o a través del aprendizaje autónomo y la capacitación, cuya valoración se subordina a su utilidad, en el sentido de que pueda ser ser aplicada en el aula.

En síntesis, en el proceso de construcción de la identidad profesional, la identificación con un otro que modeliza la profesión y la propia experimentación del rol en ámbitos reales y concretos, generan las condiciones para la adquisición de una identidad profesional. Identidad que se nutre del oficio de los otros y de sí mismo aunque no es posible dejar de reconocer que es favorecida por una formación sistemática. Los mandatos que los "otros significativos" delegan en ellos y las culturas profesionales e institucionales transmitidas en el ejercicio profesional, permiten que el profesor pueda asumir el rol dándole cierto sentido.

\section{Conclusiones}

Analizar los aspectos que componen el sí mismo profesional y sus orígenes, da cuenta del entramado individual y social, espacial y temporal del proceso de construcción de la identidad profesional. Proceso complejo en el que se van manifestando las continuidades que otorgan sentido de mismidad en las representaciones de sí mismos que tienen los profesores de secundaria.

Retomando los interrogantes que orientaron nuestras reflexiones, encontramos que son componentes configuradores de la identidad profesional de estos docentes: la vocación, el placer o gusto de enseñar y el compromiso con el trabajo; la disciplina que enseñan; la socialización de los adolescentes a través de la formación personal integral. A éstos se agregan ciertas condiciones de la organización del sistema educativo: el nivel medio y la acreditación profesional, que les permiten insertarse socialmente con cierto status que legitima el rol y la función sociales.

Esas representaciones fueron tomando forma a través de experiencias personales, académicas y laborales. El retorno al pasado a través de la narración de las biografías profesionales nos permite comprender cómo en la actualidad se ponen en tensión e interpelan algunos de los sentidos asumidos amenazando su continuidad. Los profesores y la identidad que han construido no escapan a las circunstancias que estamos viviendo en relación con la crisis de sentido de la institución en donde se inscriben: la Escuela Media; fenómeno que se enmarca en procesos sociales, políticos, culturales y educativos que caracterizan nuestro tiempo. Al respecto, identificamos aquí algunas tensiones, dilemas u oposiciones que, desde las percepciones de los docentes, atraviesan su quehacer cotidiano. Ellas posibilitan mostrar cómo los fundamentos de la identidad construida se han ido erosionando.

Por ejemplo, a la definición del rol docente en tanto formador se opone la necesidad urgente de cumplir otros roles para suplir demandas de los alumnos aunque sean responsabilidades de otras instituciones: 
"con los chicos somos psicólogos, madre, padre, tenés que hablarles, acá es muy distinto, ...hay que dejar de lado los contenidos y hablarles..." (S).

También esa imagen del profesor como modelo o referente para los jóvenes, tensiona con la imagen que dan algunos docentes que descuidan su presencia, el vocabulario frente a los alumnos y sus obligaciones laborales.

Al ideal de formación de excelencia académica forjado en las propias experiencias y en los modelos de docentes, se contrapone "un mandato de retención" de los alumnos en el sistema, al margen de la calidad y relevancia de sus aprendizajes ${ }^{8}$.

A la definición de la docencia entendida como "apostolado", con una fuerte valoración de la vocación y del compromiso con la tarea, se opone la idea de la docencia como un medio para otro fin. Como lo expresa una profesora:

“... la docencia se convierte en una salida laboral, no en una vocación, yo creo que ahí radica el problema fundamental, porque creo que hay cosas que uno hace que van mas allá de lo laboral, cuando vos realmente amas hacer una cosa..." (ME).

Estas oposiciones que aparecen en el discurso de los profesores refleja las escisiones existentes en el colectivo y la cultura profesional, por lo que no es posible componer una noción de colectivo homogéneo conforme a una idea esencial y común de la docencia de nivel medio (Lang, 2005). Al respecto, Tedesco relaciona la ruptura de la cohesión interna del cuerpo docente con la ausencia de una visión hegemónica acerca del papel de la escuela y de los docentes. "La narrativa tradicional sobre la cual se construyó la identidad docente está hoy en crisis, pero no aparece una nueva con similar fuerza hegemónica" (Tedesco, en Tenti, ob.cit.)

En este escenario que interpela la identidad de las instituciones educativas y de los sujetos que trabajan en ellas, los profesores entrevistados se definen respecto de una figura idealizada del docente. Posiblemente, frente al riesgo de desplazamiento de la especificidad, los profesores intenten sostener ciertas tradiciones para mantener la continuidad de su identidad profesional. Entendiendo en este caso a las tradiciones como "...prácticas que "inventa" el hombre ante la necesidad de construir un soporte "temporal" que garantice la permanencia del pasado en el presente $y$, por ende, una continuidad de la cual sostenerse" (Fattore, N en Baquero, R; Diker, G. y Frigerio, G, 2007: 14). En este sentido, inferimos que de alguna manera los profesores aspiran mantener la mismidad y continuidad de una identidad y de una tarea interpelada pero no desarticulada.

\section{Bibliografía}

Abraham, A. (1998) "La identidad profesional de los docentes y sus vicisitudes" en Revista del Instituto de Investigaciones en Ciencias de la Educación. Año VII № 13.Buenos Aires: Miño y Dávila editores: 10 - 14 .

Berger, P. y Luckman, T (1991) La construcción social de la realidad. Bs. As.: Amorrortu.

\footnotetext{
${ }^{8}$ Desarrollamos con mayor profundidad esta cuestión en un trabajo anterior denominado "Los profesores frente a las demandas sociales de inclusión, retención y contención en la Escuela Media. Análisis de una problemática de la institución educativa y del trabajo del profesor en nuestra región". Ponencia presentada en las Jornadas de Investigaciones en Estudios Sociales del Centro de Estudios Sociales de la UNNE. 2007
} 
Bolivar, A. (Dir) (1999) Profissao Profesor. O itinerario profissional e a construçao da escola. Brasil: EDUSC Editora da Universidade do Sagrado Coraçao.

Davini, M. C. (1995) La Formación Docente en cuestión: política y pedagogía. Buenos Aires. Paidos.

Diker, G. y Terigi, F. (1997) La formación de maestros y profesores: hoja de ruta. Buenos Aires. Paidos.

Dubet, F y Martuccelli, D. (1998) En la escuela. Sociología de la experiencia escolar. España: Losada.

Fattore, Natalia. (2007) "Apuntes sobre la forma escolar "tradicional" y sus desplazamientos". En Baquero, R; Diker, G. y Frigerio, G (comps) Las formas de lo escolar. Bs As: del estante editorial.

Ferry, G. (1997) Pedagogía de la formación. Novedades Educativas, Bs.As.

Kornblit, A. L. (coord.) (2004). Metodologías cualitativas en ciencias sociales: modelos y procedimientos de análisis. Buenos Aires: Biblos.

Lang, V. (2005) "La construcción social de las identidades profesionales de los docentes en Francia", en Seminario Internacional "La renovación del oficio docente: vocación, trabajo y profesión en el siglo XXI". Bs. As.: IIPE-UNESCO.

Rivas, J. I., Sepúlveda, M. P., Rodrigo, P. M., Alonso, M., Cortés, C., Gimeno, V., et al. (2000). El trabajo de los docentes de secundaria: estudio biográfico de su cultura profesional. En Revista Interuniversitaria de Formación del Profesorado. № 39. España: Asociación Universitaria de Formación del Profesorado. 133146.

Tenti Fanfani, E. (2005). "Conferencia Inaugural". En Seminario Internacional "La renovación del oficio del docente. Vocación, trabajo y profesión en el siglo XXI". Bs As: IIPE-UNESCO. Mimeo.

Tenti Fanfani, E. (2005): La condición docente. Análisis comparado de la Argentina, Brasil, Perú y Uruguay. Bs.As. Siglo XXI Editores.

Veiravé, D.; Ojeda, M.; Núñez, C. y Delgado, P. (2006) "Las narrativas biográficas para la comprensión de la vida y el trabajo del profesor de escuela media. Análisis sobre las dimensiones y los planos de trabajo metodológico". En VI SEMINÁRIO DA REDESTRADO - Regulação Educacionale Trabalho Docente. Rio de Janeiro: UERJ - REDESTRADO. Publicación en formato digital.

Veiravé, D.; Ojeda, M.; y Delgado, P. (2007): "Los profesores frente a las demandas sociales de inclusión, retención y contención en la Escuela Media. Análisis de una problemática de la institución educativa y del trabajo del profesor en nuestra región". En Pérez, A. M. y Benitez, M.A. (comp.) Coloquio de Estudios Sociales. Corrientes, Centro de Estudios Sociales de la UNNE: Moglia Ediciones.

Veiravé, D; Ojeda, M; Delgado, P; Núñez, C y Amud, C. (2008) "Nuevas configuraciones del trabajo docente del nivel medio. Prácticas dispersas, diversas y a la intemperie". En VII Seminario de REDESTRADO "Nuevas Regulaciones en América Latina". Bs As: UBA - REDESTRADO. Publicación en formato digital. 